\title{
STRONGLY EXPOSED POINTS IN THE UNIT BALL OF TRACE-CLASS OPERATORS
}

\author{
KOUROSH NOUROUZI
}

Received 23 April 2001 and in revised form 22 September 2001

\begin{abstract}
A theorem of Arazy shows that every extreme point of the unit ball of trace-class operators is strongly exposed. We give this result a simpler and direct proof here.
\end{abstract}

2000 Mathematics Subject Classification: 46A55, 47B20.

The main purpose of this paper is to give a direct proof of an interesting special case of a far-reaching theorem of Arazy [1, 2].

Arazy studied the extreme, exposed, and strongly exposed points in the unit balls of symmetrically normed ideals (of operators) acting on a separable Hilbert space, and he obtained very useful and complete results in [2] on the extremal structure of these operator balls. Arazy's study of symmetrically normed ideals is, on the one hand, quite general. On the other hand, the ideal of trace-class operators is, for a variety of reasons, perhaps the most interesting of the symmetrically normed ideals. Because of the importance of the trace-class operators, and in the light of sustained interest in exposed points amongst Banach space theorists, we thought it is worthwhile to find a fairly direct proof of Arazy's theorem in this particular case. Our proof, presented herein, is straightforward in the sense that it relies essentially only on fundamental properties of singular values, as explained in the monograph of Gohberg and Kreĭn [3].

THEOREM 1 (Arazy). Let $\Im_{1}$ denote the Banach space of the trace-class operators acting on an infinite-dimensional separable complex Hilbert space, and assume that

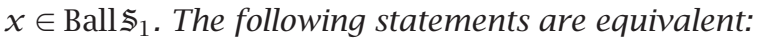

(a) $x$ has rank 1 and $\operatorname{tr}\left(x^{*} x\right)=1$;

(b) $x$ is an extreme point of Ball $\Im_{1}$;

(c) $x$ is a strongly exposed point of Ball $\Im_{1}$.

The equivalence of (a) and (b) seems to have first been determined by Holub in [5]. Before moving to the proof, the relevant definitions are reviewed below.

An element $\omega$ in a convex set $C$ in a complex Banach space $X$ is an extreme point of $C$ if the equation $\omega=t \omega_{1}+(1-t) \omega_{2}$, for $t \in(0,1) \subset \mathbb{R}$ and $\omega_{1}, \omega_{2} \in C$, is satisfied only with $\omega_{1}=\omega_{2}=\omega$. A point $\omega \in C$ is strongly exposed if there is a continuous linear function $f: X \rightarrow \mathbb{C}$ such that

(i) $\operatorname{Re} f(\gamma)<\operatorname{Re} f(\omega)$, for all $\gamma \in C \backslash\{\omega\}$,

(ii) $\operatorname{Re} f\left(\gamma_{k}\right) \rightarrow \operatorname{Re} f(\omega)$, for a sequence, $\left\{\gamma_{k}\right\}_{k} \subset C$, only if $\gamma_{k} \rightarrow \omega$.

If only (i) holds for $\omega \in C$, then $\omega$ is said to be an exposed point of $C$. Exposed points of $C$ (if they exist) are extreme points of $C$, but in general extreme points need not be exposed. 
For any Banach space $X$, let

(1) Ball $X=\{x \in X:\|x\| \leq 1\}$,

(2) ext Ball $X=\{x \in$ Ball $X: x$ is an extreme point of Ball $X\}$.

It is an elementary consequence of the Cauchy-Schwarz inequality that the extreme points of Ball $H$ in any Hilbert space $H$ (of any dimension) are strongly exposed. In contrast, if $B(H)$ denotes the algebra of a bounded operator acting on a Hilbert space $H$, then the extreme points of Ball $B(H)$ are exposed if and only if $H$ is separable, and they are strongly exposed if and only if $H$ is finite dimensional. (These results were proved by Grzaślewicz [4].) Arazy's work provides a complete analysis of the situation concerning strongly exposed points in the unit balls of symmetrically normed ideals.

Henceforth, $H$ will denote a separable, infinite-dimensional Hilbert space; $B(H)$ is the $C^{*}$-algebra of (bounded) operators acting on $H$; and $K(H)$ denotes the ideal of compact operators acting on $H$. If $x \in B(H)$, then $x$ has a polar decomposition $x=$ $w|x|$, where $|x|$ is the (unique) positive square root of $x^{*} x$ and where $w$ is the (unique) partial isometry whose initial space is the closure of the range of $|x|$ and whose final space is the closure of the range of $x$.

For $x \in K(H)$, the singular values of $x$ are the elements $s_{k}(x)$ of the decreasing sequence $\left\{s_{k}(x)\right\}_{k \in \mathbb{Z}^{+}}$of nonnegative real numbers $s_{k}(x)=\sqrt{\lambda_{k}\left(x^{*} x\right)}$, where

$$
\lambda_{k}\left(x^{*} x\right)=\min \left\{\max \left\{\|x \xi\|^{2}: \xi \in L^{\perp},\|\xi\|=1\right\}: L \subset H, \operatorname{dim} L=k-1\right\} .
$$

Let $\|\cdot\|$ denote the operator norm on $B(H)$, namely, $\|x\|=\sup \{\|x \xi\|: \xi \in H$, $\|\xi\|=1\}$. If $x \in K(H)$, then, by [3, page 29],

$$
\begin{gathered}
s_{1}(x)=\|x\| ; \\
s_{n}(x)=\min \{\|z-x\|: \operatorname{rank} z \leq n-1\}, \quad \text { for } n \geq 2 .
\end{gathered}
$$

The trace class is the ideal set $\Im_{1}$ of $B(H)$, defined by $\Im_{1}=\left\{x \in K(H): \sum_{n} s_{n}(x)<\right.$ $\infty$, is an ideal of $B(H)$, and the function $\|\cdot\|_{1}: \Im_{1} \rightarrow \mathbb{R}_{0}^{+}$, given by

$$
\|x\|_{1}=\sum_{n=1}^{\infty} s_{n}(x), \quad x \in \Im_{1},
$$

is a norm on $\Im_{1}$ under which $\Im_{1}$ is a Banach space.

For every $x \in \Im_{1}, \sum_{n}\left\langle x \phi_{n}, \phi_{n}\right\rangle$, where $\left\{\phi_{n}\right\}_{n \in \mathbb{Z}^{+}}$is an orthonormal basis of $H$ is absolutely convergent. This defines a linear functional on $\Im_{1}$ called the trace

$$
\operatorname{tr}(x)=\sum_{n=1}^{\infty}\left\langle x \phi_{n}, \phi_{n}\right\rangle, \quad x \in \mathfrak{S}_{1} .
$$

It is well known that the definition of the trace is independent of the choice of orthonormal basis.

If $x \in \Im_{1}$, then let $\vec{x}$ be the vector in $\ell_{1}$ whose $n$th component is $s_{n}(x)$. It is clear from the definition of $\Im_{1}$ that $x \in$ Ball $\Im_{1}$ if and only if $\vec{x} \in$ Ball $\ell_{1}$.

Proof of Arazy's THeOrem. We show that $(b) \Rightarrow(a) \Rightarrow(c) \Rightarrow(b)$.

Thus, assume that $x \in$ extBall $\Im_{1}$, and consider $\vec{x} \in$ Ball $\ell_{1}$. We aim to show that $\vec{x}$ is an extreme point of Ball $\ell_{1}$. Suppose that $\vec{x}=(1 / 2) \alpha+(1 / 2) \beta$, for some $\alpha, \beta \in$ Ball $\ell_{1}$. 
If $x=w|x|$ is the polar decomposition of $x$, where $|x|$ has the spectral decomposition $|x| \xi=\sum_{n} s_{n}(x)\left\langle\xi, \phi_{n}\right\rangle \phi_{n}$, for $\xi \in H$. Let $a, b \in B(H)$ be defined so that the action of $a$ and $b$ on each $\xi \in H$ is

$$
\sum_{n=1}^{v} \alpha_{n}\left\langle\xi, \phi_{n}\right\rangle w \phi_{n}, \quad \sum_{n=1}^{v} \beta_{n}\left\langle\xi, \phi_{n}\right\rangle w \phi_{n},
$$

where $\alpha_{n}$ and $\beta_{n}$ denote the $n$th components of the vectors $\alpha, \beta \in \ell_{1}$ and $\nu \in \mathbb{Z}^{+} \cup\{\infty\}$. Thus, $x=(1 / 2) a+(1 / 2) b$. Because $\|a\|_{1} \leq\|w\|\left(\sum_{n}\left|\alpha_{n}\right|\right) \leq 1$ and, likewise, $\|b\|_{1} \leq 1$, $x$ is an average of two elements (namely, $a$ and $b$ ) from the unit ball of $\Im_{1}$. Hence, $a=b=x$.

The projection $w^{*} w$ has the range $\left(\operatorname{Span}\left\{\phi_{n}\right\}_{n=1}^{v}\right)^{-}$, and therefore $w^{*} w \phi_{n}=\phi_{n}$, for all $n$. Thus, for all $\xi \in H$,

$$
|x| \xi=w^{*} x \xi=w^{*} a \xi=\sum_{n=1}^{v} \alpha_{n}\left\langle\xi, \phi_{n}\right\rangle \phi_{n},
$$

which means that $\alpha_{n}=s_{n}(x)$ for every $n$. Similarly, $\beta_{n}=s_{n}(x)$. Hence, $\alpha=\beta=\vec{x}$, which proves that $\vec{x} \in$ ext Ball $\ell_{1}$.

Now let $e_{n} \in \ell_{1}$ be the vector with the real number 1 in position $n$ and zero in all other positions. Because extBall $\ell_{1}=\left\{-e_{n}, e_{n}: n \in \mathbb{Z}^{+}\right\}, x \in$ extBall $\Im_{1}$ if and only if $x$ has exactly one nonzero singular value, namely $s_{1}(x)$, and $s_{1}(x)=1$. In other words, $\|x\|^{2}=\operatorname{tr}\left(x^{*} x\right)=\operatorname{rank} x=1$, completing the proof that $(\mathrm{b}) \Rightarrow(\mathrm{a})$.

To prove that $(\mathrm{a}) \Rightarrow(\mathrm{c})$, let $x$ be a rank-1 operator of norm 1. From the polar decomposition $x=w|x|$ of $x$, there are unit vectors $\phi_{1}, \psi_{1} \in H$ such that $x \xi=\left\langle\xi, \phi_{1}\right\rangle \psi_{1}$, for every $\xi \in H$, where $w \phi_{1}=\psi_{1}$ and $w w^{*}$ is the projection onto $\operatorname{Span}\left\{\psi_{1}\right\}$.

Set $p=|x|$. Because $x$ is a rank-1 operator of norm $1, p$ is a rank-1 projection whose range is spanned by $\phi_{1}$. Extend the singleton set $\left\{\phi_{1}\right\}$ to an orthonormal basis $\left\{\phi_{n}\right\}_{n \in \mathbb{Z}^{+}}$of $H$. Thus, the trace of every $z \in \mathfrak{S}_{1}$ is given by $\operatorname{tr}(z)=\sum_{n}\left\langle z \phi_{n}, \phi_{n}\right\rangle$.

Define a linear functional $f$ on $\Im_{1}$ by

$$
f(y)=\operatorname{tr}\left(p w^{*} y p\right), \quad \forall y \in \mathfrak{s}_{1} .
$$

Then $f(x)=\operatorname{tr}\left(p w^{*} w|x| p\right)=\operatorname{tr}(p)=1$, because $p$ is a rank-1 projection. So, $\operatorname{Re} f(x)=$ $f(x)=1$. Moreover, if $y \in$ Ball $\lessgtr_{1}$, then

$$
|f(y)|=\left|\left\langle w^{*} y \phi_{1}, \phi_{1}\right\rangle\right| \leq\left\|w^{*}\right\|\|y\|=s_{1}(y) \leq \sum_{n=1}^{\infty} s_{n}(y)=\|y\|_{1} \leq 1 .
$$

Thus, $f$ is a support functional for Ball $\Im_{1}$.

Assume that $\left\{y_{k}\right\}_{k} \subset$ Ball $\Im_{1}$ and $\lim _{k} \operatorname{Re} f\left(y_{k}\right)=\operatorname{Re} f(x)=f(x)=1$, that is,

$$
\lim _{k} \operatorname{Re}\left\langle w^{*} y_{k} \phi_{1}, \phi_{1}\right\rangle=1
$$

Then, by (8), the sequence $\left\{y_{k}\right\}_{k}$ has the property that, for all $n \geq 2$,

$$
\lim _{k} s_{1}\left(y_{k}\right)=\lim _{k}\left\|y_{k}\right\|=\lim \left\|y_{k}\right\|_{1}=1, \quad \lim _{k} s_{n}\left(y_{k}\right)=0 .
$$


Furthermore,

$$
\begin{aligned}
\left\|y_{k} \phi_{1}-x \phi_{1}\right\|^{2} & =\left\|y_{k} \phi_{1}\right\|^{2}-2 \operatorname{Re}\left\langle y_{k} \phi_{1}, x \phi_{1}\right\rangle+\left\|x \phi_{1}\right\|^{2} \\
& =\left\|y_{k} \phi_{1}\right\|^{2}-2 \operatorname{Re}\left\langle y_{k} \phi_{1}, w \phi_{1}\right\rangle+\left\|\psi_{1}\right\|^{2} \\
& \leq\left\|y_{k}\right\|^{2}\left\|\phi_{1}\right\|^{2}-2 \operatorname{Re}\left\langle w^{*} y_{k} \phi_{1}, \phi_{1}\right\rangle+\left\|\psi_{1}\right\|^{2} \\
& \leq 2\left(1-\operatorname{Re}\left\langle w^{*} y_{k} \phi_{1}, \phi_{1}\right\rangle\right) .
\end{aligned}
$$

Thus, by (9),

$$
\left\|y_{k} \phi_{1}-x \phi_{1}\right\| \longrightarrow 0
$$

Because $x$ is a rank-1 operator, the inequalities in [3, page 29] are

$$
s_{n+1}\left(y_{k}\right) \leq s_{n}\left(y_{k}-x\right) \leq s_{n-1}\left(y_{k}\right), \text { for } n \geq 2 .
$$

Hence, for every $k$,

$$
\begin{aligned}
\left\|y_{k}-x\right\|_{1} & =s_{1}\left(y_{k}-x\right)+s_{2}\left(y_{k}-x\right)+\sum_{n=3}^{\infty} s_{n}\left(y_{k}-x\right) \\
& \leq 2 s_{1}\left(y_{k}-x\right)+\sum_{n=2}^{\infty} s_{n}\left(y_{k}\right) \\
& =2\left\|y_{k}-x\right\|+\left\|y_{k}\right\|_{1}-s_{1}\left(y_{k}\right) \\
& \leq 2\left\|y_{k}-x\right\|+1-s_{1}\left(y_{k}\right) .
\end{aligned}
$$

Therefore, to prove that $\lim _{k} y_{k}=x$ in $\mathfrak{\Im}_{1}$ it is sufficient, by (10), to prove that $\left\|y_{k}-x\right\| \rightarrow 0$.

The singular value $s_{2}\left(y_{k}\right)$ measures the distance in $B(H)$ from $y_{k}$ to the set of operators whose rank is at most 1 . Thus, by (10), there is a sequence $\left\{z_{k}\right\} \in \Im_{1}$ of operators such that each $z_{k}$ is zero or rank-1 and $\left\|y_{k}-z_{k}\right\| \rightarrow 0$ as $k \rightarrow \infty$. From $\left\|y_{k}-x\right\| \leq\left\|y_{k}-z_{k}\right\|+\left\|z_{k}-x\right\|$ we see that it is now enough to prove that $\left\|z_{k}-x\right\| \rightarrow 0$.

Because $\left\|z_{k} \phi_{1}-x \phi_{1}\right\| \leq\left\|z_{k}-y_{k}\right\|\left\|\phi_{1}\right\|+\left\|y_{k} \phi_{1}-x \phi_{1}\right\|$, we have that $\| z_{k} \phi_{1}-$ $x \phi_{1} \| \rightarrow 0$. Hence, there exists $N \in \mathbb{Z}^{+}$such that $z_{k} \phi_{1} \neq 0$, for all $k \geq N$. In all cases for which $z_{k} \phi_{1} \neq 0$, the vector $z_{k} \phi_{1}$ spans the range of $z_{k}$ and, therefore, there exist vectors $\eta_{k} \in H$ such that, for every $\xi \in H$,

$$
z_{k} \xi=\left\langle\xi, \eta_{k}\right\rangle z_{k} \phi_{1}
$$

Formula (15) also holds for all $k$ for which $z_{k} \phi_{1}=0$ by simply choosing $\eta_{k}=0$.

If $k \geq N$ then $z_{k} \phi_{1}=\left\langle\phi_{1}, \eta_{k}\right\rangle z_{k} \phi_{1}$; that is,

$$
\left\langle\phi_{1}, \eta_{k}\right\rangle=1, \quad \forall k \geq N .
$$

Also,

$$
||\left|y_{k}\|-\| z_{k}\|\mid \leq\| y_{k}-z_{k}\|, \quad\| z_{k}\|=\| \eta_{k}\|\| z_{k} \phi_{1} \| .\right.
$$


Therefore, the sequence $\left\{\left\|\eta_{k}\right\|\right\}_{k}$ is bounded and

$$
\lim _{k \rightarrow \infty}\left\|\eta_{k}\right\|=\lim _{k \geq N} \frac{\left\|z_{k}\right\|}{\left\|z_{k} \phi_{1}\right\|}=1 .
$$

For any $\xi \in H$,

$$
\begin{aligned}
\left\|\left(z_{k}-x\right) \xi\right\| & =\left\|\left\langle\xi, \eta_{k}\right\rangle z_{k} \phi_{1}-\left\langle\xi, \phi_{1}\right\rangle x \phi_{1}\right\| \\
& =\left\|\left\langle\xi, \eta_{k}\right\rangle z_{k} \phi_{1}-\left\langle\xi, \eta_{k}\right\rangle x \phi_{1}+\left\langle\xi, \eta_{k}\right\rangle x \phi_{1}-\left\langle\xi, \phi_{1}\right\rangle x \phi_{1}\right\| \\
& \leq\left|\left\langle\xi, \eta_{k}\right\rangle\right|\left\|z_{k} \phi_{1}-x \phi_{1}\right\|+\left\|x \phi_{1}\right\|\left|\left\langle\xi, \eta_{k}-\phi_{1}\right\rangle\right| \\
& \leq\|\xi\|\left(\left\|\eta_{k}\right\|\left\|z_{k} \phi_{1}-x \phi_{1}\right\|+\left\|\eta_{k}-\phi_{1}\right\|\right) .
\end{aligned}
$$

Therefore, we have $\left\|z_{k}-x\right\| \rightarrow 0$ if we can prove that $\left\|\eta_{k}-\phi_{1}\right\| \rightarrow 0$. But this is so, because

$$
\begin{aligned}
\left\|\eta_{k}-\phi_{1}\right\|^{2} & =\left\|\eta_{k}\right\|^{2}-2 \operatorname{Re}\left(\left\langle\eta_{k}, \phi_{1}\right\rangle\right)+\left\|\phi_{1}\right\|^{2} \\
& =\left\|\eta_{k}\right\|^{2}-2+1=\left\|\eta_{k}\right\|^{2}-1 .
\end{aligned}
$$

Thus, from (18) we conclude that $\left\|\eta_{k}-\phi_{1}\right\| \rightarrow 0$. Hence, (8) $\Rightarrow(10)$.

The proof of $(c) \Rightarrow(b)$ is a standard argument in convexity theory, which is, therefore, omitted here.

ACKNOWLEDGments. The author wishes to express his debt to Professor Douglas R. Farenick for much more than just conversations related to the contents of this paper. This work is supported in part by the Ministry of Higher Education of Iran.

\section{REFERENCES}

[1] J. Arazy, Basic sequences, embeddings, and the uniqueness of the symmetric structure in unitary matrix spaces, J. Funct. Anal. 40 (1981), no. 3, 302-340.

[2] _ On the geometry of the unit ball of unitary matrix spaces, Integral Equations Operator Theory 4 (1981), no. 2, 151-171.

[3] I. C. Gohberg and M. G. Krein, Introduction to the Theory of Linear Nonselfadjoint Operators, translated from the Russian by A. Feinstein. Translations of Mathematical Monographs, vol. 18, American Mathematical Society, Rhode Island, 1969.

[4] R. Grzaślewicz, Exposed points of the unit ball of $\mathscr{L}(H)$, Math. Z. 193 (1986), no. 4, 595-596.

[5] J. R. Holub, On the metric geometry of ideals of operators on Hilbert space, Math. Ann. 201 (1973), 157-163.

KOURosh Nourouzi: DEPARTMENT OF MATHEMATICS, KNT UNIVERSITY OF TECHNOLOGY, 322 West Mirdamad, AVE. 19696, P.O. BoX 15875-4416, TEHRAN, IRAN 


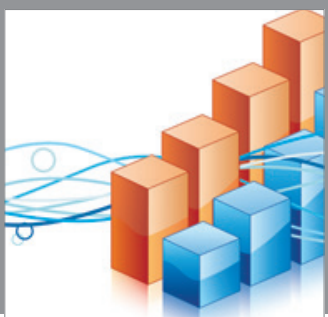

Advances in

Operations Research

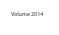

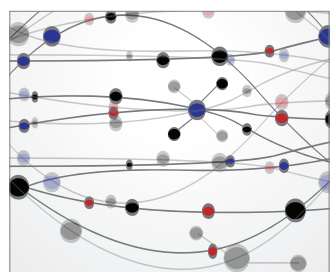

\section{The Scientific} World Journal
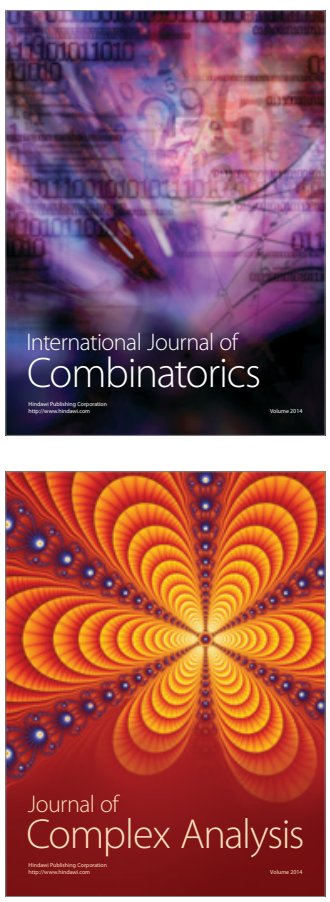

International Journal of

Mathematics and

Mathematical

Sciences
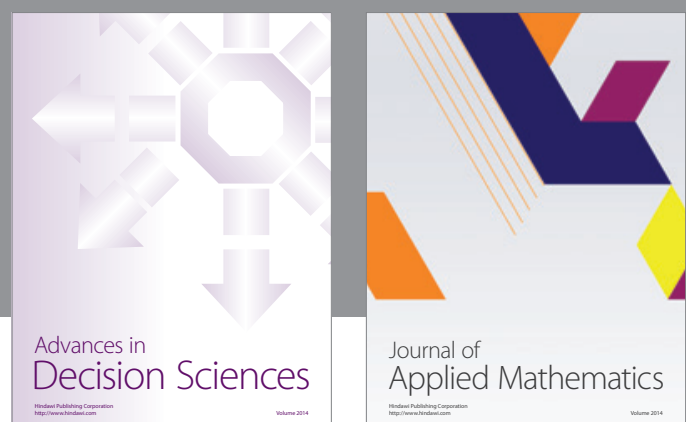

Journal of

Applied Mathematics
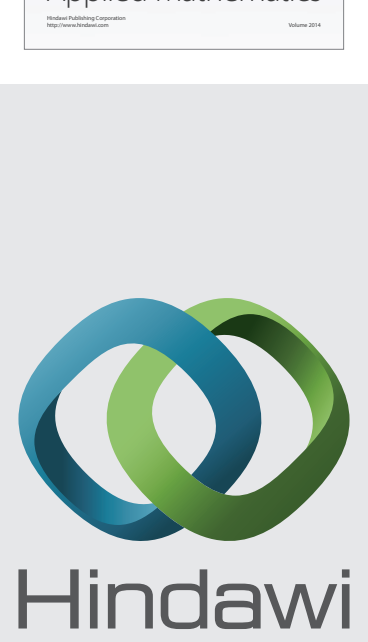

Submit your manuscripts at http://www.hindawi.com
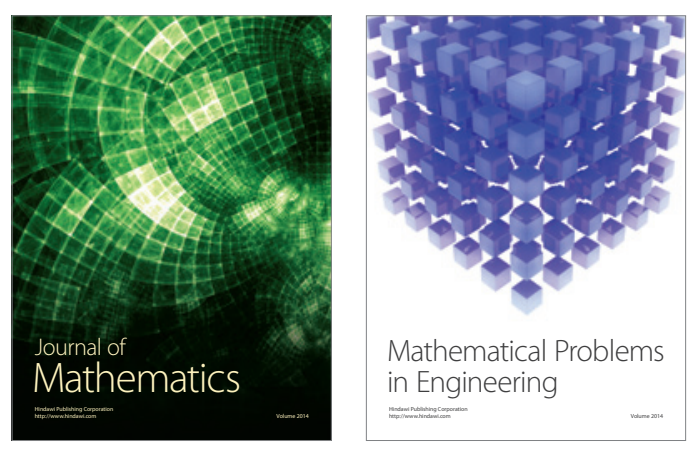

Mathematical Problems in Engineering
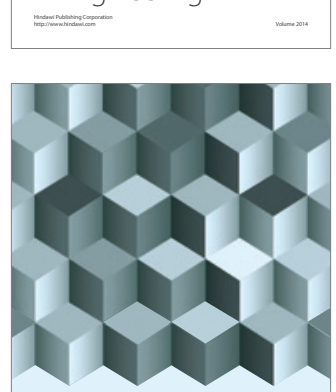

Journal of

Function Spaces
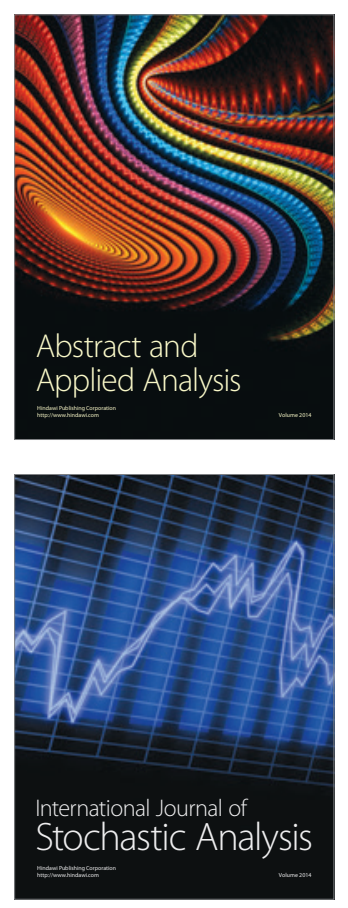

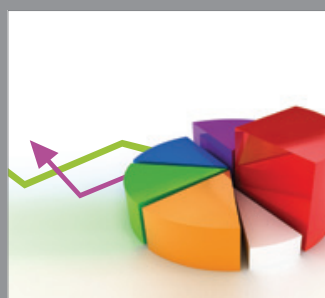

ournal of

Probability and Statistics

Promensencen
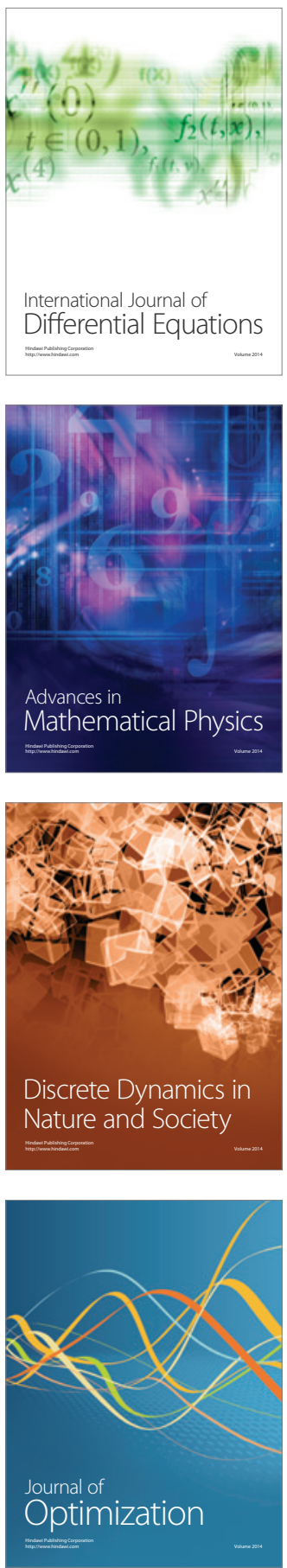